\title{
LA INVESTIGACIÓN FORMATIVA: UNA EXPERIENCIA DEL CUERPO ACADÉMICO ESTUDIOS EN EDUCACIÓN
}

\section{FORMATIVE RESEARCH: A CASE OF ACADEMIC GROUP ESTUDIOS EN EDUCACIÓN}

\author{
Hilda López-Domínguez* y Héctor Carmona-Vázquez**
}

\begin{abstract}
RESUMEN
El presente trabajo es el resultado de una investigación desarrollada con la participación del Cuerpo Académico: Estudios en Educación (CAEE) a partir de algunas entrevistas en profundidad realizadas a miembros y estudiantes participantes del mismo. La finalidad de dichas entrevistas fue rescatar, reconstruir y reflexionar en la experiencia de miembros del CA y estudiantes en cuanto a su implicación en la estrategia Investigación Formativa (IF), así también poner en relevancia el impacto que ha tenido este ejercicio tanto para con la formación de los estudiantes en su posible futura tarea como investigadores, como respecto al enriquecimiento de la formación de los investigadores de acuerdo a lo que conocen y aportan los estudiantes en una dinámica de "todos aprendemos de todos". Esto dado que se considera emergente evidenciar las experiencias de investigación que distintos grupos están teniendo en pro de difundir dichas experiencias y ponerlas como referencias para su reproducción, adaptación o exclusión según las necesidades y circunstancias de quienes participan en la investigación.
\end{abstract}

Palabras clave: investigación, formación, proceso.

\begin{abstract}
This work is the outcome of a research developed in collaboration with the Academic Group "Estudios en Educación" based on interviews conducted to students and members of the same Academic Group. The purpose for these interviews was resuming, reconstructing and reflecting on their experiences in the implication of the strategy "Formative Research" and the impact that this exercise has had in the professional training of students as possible future researchers, as well as in the improvements in the professional training of researchers regarding the student's knowledge and their contributions in an exercise of "Everyone learn from everyone". This is considered as emerging research experiences show that different groups are taking towards these experiences and disseminate them as references for reproduction, adaptation or exclusion according to the needs and circumstances of those involved in the investigation.
\end{abstract}

Keywords: research, process, research, formation.

Fecha de recepción: Marzo 24 de 2015 / Fecha de aceptación: Mayo 06 de 2015

Tipología: Artículo de Investigación Científica y Tecnológica

Para citar este artículo: López, D. H., \& Carmona, V. H. (2015. La investigación formativa: una experiencia del cuerpo académico estudios en educación. Praxis. Vol. 11, 47 - 53

\footnotetext{
*Maestra en Investigación en Psicología Aplicada a la Educación, Universidad Veracruzana. México. Email: hlopezdominguez@gmail.com

** Lic. en Antropología y Maestro en Didáctica del Francés, Universidad Veracruzana. México. Email: heccar.vz@gmail.com
} 


\section{INTRODUCCIÓN}

En los últimos años se ha puesto especial interés en el trabajo colaborativo. Específicamente dada la influencia que el enfoque constructivista ha tenido en el nuevo concepto de enseñanzaaprendizaje. Docentes y alumnos hemos tenido que ajustarnos a una nueva dinámica de trabajo, aprendizaje, enseñanza, convivencia, entre otras.

Esta nueva dinámica que hoy intenta florecer es una iniciativa de los grupos de investigación a partir de integrar a personas formadas desde distintas disciplinas, condición que enriquece la labor del propio grupo y a su vez, le permite a alumnos practicar, aplicar y reflexionar lo aprendido a nivel teórico.

En consecuencia, ante los nuevos escenarios: sociales, culturales, económicos y políticos, las universidades han tenido que encararlos efectivamente, a través de estrategias centradas en sus funciones sustantivas: la docencia, la investigación, la extensión y la difusión de la cultura. Es la segunda función sustantiva de la Universidad Veracruzana en la que se enfatiza en este trabajo. Esto dado que coadyuva en la construcción, creación y recreación del conocimiento así como también representa el nivel intelectual y científico de la sociedad. Hacer investigación permite conocer problemáticas existentes, al tiempo que posibilita buscar soluciones. Es decir, la investigación se propone ir de un estado de insatisfacción a uno de satisfacción.

Es así que, para su fortalecimiento, las universidades junto con las políticas institucionales han adoptado diversas medidas, entre ellas destaca el trabajo colectivo, mismo que reafirma la idea de conjuntar esfuerzos, imaginarios, recursos. Tales medidas condujeron nuestra mirada hacia la estrategia que los investigadores del Cuerpo Académico Estudios en Educación (CAEE), dada su experiencia, necesidades, circunstancias y recursos, ponen en práctica a fin de cumplir con aquello que hoy resulta emergente, el trabajo colaborativo. La finalidad de este trabajo es construir un concepto de investigación formativa gestado desde la experiencia del CAEE tomando en cuenta una descripción de los elementos de organización y comunicación en grupo, así como aquellos de carácter formativo implicados en su práctica.

A continuación se presenta parte del reporte de un estudio realizado, tratando de difundir una experiencia de investigación que bien podría añadirse a aquellas adoptadas por otros grupos de investigación. Con la presente pretendemos advertir la necesidad de beneficiar a más alumnos, permitiéndoles tener una experiencia de investigación antes de estar en la posibilidad de ejercerla de manera formal.

El estudio se justifica en la idea de que la investigación educativa es un campo emergente en el que debemos profundizar para conocer las dificultades, logros y alcances y en consecuencia, conozcamos y reflexionemos en los elementos que han permitido resolver problemáticas educativas. Por tanto, abordar la investigación formativa permite reconocerla por su impacto, ponerla de manifiesto como una de las estrategias de las que se ha valido el CAEE a fin de sacar adelante las múltiples actividades que desarrolla y exponerla como una "posibilidad" en la que otros grupos de investigación participen, la modifiquen, la reestructuren y la caractericen según sus necesidades con la finalidad de obtener los resultados favorables para todos los miembros del grupo poniendo énfasis en la formación permanente de los mismos.

\section{METODOLOGÍA}

Este trabajo profundiza en los elementos que caracterizan a la investigación formativa desde la experiencia del Cuerpo Académico Estudios en Educación (CAEE). Dicha caracterización fue posible a partir de entrevistar a dos miembros del Cuerpo Académico y a dos estudiantes que colaboraban en el mismo. Se realizaron entrevistas a profundidad basadas en un guión semiestructurado, dicho guión fue estructurado de forma distinta para estudiantes y para miembros del CA en tanto sus experiencias difieren dado el tipo de participación que tiene cada uno de ellos.

La investigación estuvo fundamentada en el método biográfico narrativo con caso único, el 
cual argumenta en pro de construir y reconstruir voces, darles la importancia y el valor que se merecen, dado que son informantes directos. Este método dentro del área de la investigación educativa permite rescatar lo que diversos autores sustentan, las voces de los propios implicados, lo que generará un conocimiento de fondo, capaz de dar a conocer en detalle los tropiezos, alcances y dificultades con los que se han enfrentado los miembros del CAEE.

A partir de reconstruir la experiencia de los miembros del CAEE y conjuntarla con aquella vivida por los estudiantes, se construyó un concepto propio de la investigación formativa. Lo anterior tuvo lugar bajo las siguiente preguntas de investigación: ¿Cómo caracteriza el Cuerpo Académico a la Investigación Formativa desde su práctica?, ¿qué condiciones posibilitan u obstruyen la implementación de la estrategia IF?, ¿cómo impacta esta estrategia de investigación en la formación del CAEE?

\section{RESULTADOS Y DISCUSIÓN}

A partir de las entrevistas realizadas se rescataron los elementos implicados en la IF situándonos desde la experiencia de los actores involucrados. Este proceso nos permitió conocer cómo aplican y vivencian la implementación de la estrategia en su labor investigativa. Los elementos que se consideraron para la reconstrucción de la experiencia con IF y posteriormente para la conceptualización y caracterización de dicha estrategia son: trabajo colectivo-colaborativo (TCC); los estudiantes también colaboran; las tareas; la organización en el TCC; la comunicación en el TCC; alcances y dificultades; actividades detonantes en la práctica del CAEE; limitaciones infraestructurales y administrativas; las relaciones interpersonales y por último, el impacto profesional y personal de la experiencia de los estudiantes.

En el cuerpo de la investigación se describieron, analizaron y sistematizaron todas y cada una de las variables pero, para efecto de este documento hacemos referencia sólo al concepto investigación formativa que a continuación se explicita.

\section{Investigación formativa}

La investigación formativa recibe este nombre debido a que es una estrategia para la construcción de conocimientos que sigue los pasos propios del proceso investigativo, con la diferencia de incluir en su práctica actores de diversas disciplinas y grados de formación, lo cual enriquece y fortalece al mismo tiempo, la ya mencionada construcción de conocimientos. Su práctica conlleva una toma de conciencia sobre el proceso investigativo y fomenta que, como parte de ésta, se sumerja y tome un ritmo de trabajo e involucramiento cada vez mayor o más profundo, caracterizada ésta última por su creatividad, innovación, por el uso de métodos rigurosos, validación y juicio crítico de pares, entre otras características. Constituye la aplicación de hallazgos obtenidos durante el proceso para afinar y mejorar mientras se trabaja, y para servir a los involucrados como medio de reflexión y aprendizaje, incluye un seguimiento de actitudes, creencias, respuestas, asimilación de conocimientos y destrezas al momento de ser ejecutada.

En consecuencia, la IF deriva en posibilidades para transformar la práctica de la investigación, desarrolla habilidades cognoscitivas: análisis, pensamiento productivo y solución de problemas al tiempo que familiariza a estudiantes con las etapas de investigación y el planteamiento de problemas; permite un intercambio de saberes no sólo teóricos, sino también actitudinales, procedimentales, estratégicos, entre otros.

Al mismo tiempo, la estrategia atiende, en alguna medida, a los problemas que autores como Eguinoa (2006) señalan cuando habla del alumno como espectador del conocimiento, conoce del paso "a" al " $z$ " en la investigación, pero nunca ha hecho una. Esta situación se agudiza cuando los alumnos optan por titularse por una vía distinta a la elaboración de un documento recepcional (promedio, CENEVAL, grado Maestría, entre otros).

Como ya se ha mencionado, se hizo un análisis a partir de diversas elementos constitutivos de 
la investigación formativa (TCC); los estudiantes también colaboran; las tareas; la organización en el TCC; la comunicación en el TCC; alcances y dificultades; actividades detonantes en la práctica del CAEE; limitaciones infraestructurales y administrativas; las relaciones interpersonales y por último, el impacto profesional y personal de la experiencia de los estudiantes, sin embargo para efectos del presente texto nos apoyaremos en uno de los elementos que la caracterizan, "Los estudiantes también colaboran".

Al respecto, en este apartado se da constancia de la participación por parte de los alumnos, sea como becarios-tesistas, servidores sociales y/o como participantes en los seminarios que se organizan como parte de la preocupación por la actualización y formación permanente de los miembros del CAEE. Siendo éste un elemento importante de la IF.

Lo anterior adquiere relevancia cuando analizamos que el integrar en el trabajo académico a estudiantes y/o colaboradores externos es un punto que podría interpretarse desde diferentes perspectivas. A decir de los miembros del CAEE, ésta representa una "peculiaridad dado que desde sus inicios se ha integrado a estudiantes, a becarios, prestadores de servicio social” (A2). Lo anterior ha sido valorado por los alumnos en tanto no sólo se trata de incluir, sino que además va acompañada de apertura para el diálogo, de darles la oportunidad de participar activamente, pues en contra de esta apertura hallamos que en algunas situaciones "si hay estudiantes... sólo son así los que van por el café, los que hacen nada más eso, sin posibilidad de que ellos planteen también elementos importantes que puedan apoyar todo lo académico" (E1) y aunque administrativamente pueda parecer que están dando oportunidad a los jóvenes, no están formándolos.

Por tanto, es una base de la IF tener claro que cuando un grupo de investigación se conforma el trabajo que se ejecuta sea precisamente como grupo y no como un subconjunto de tareas que al juntarse construyen "una”. Se trata por tanto de que la aportación de cada uno persiga un bien común al mismo tiempo que posibilite el enriquecimiento y formación de cada miembro de dicho grupo. Es decir, la heterogeneidad de ideas, el grado de habilitación académica, los campos disciplinares y otros factores más diversifican al mismo tiempo que enriquecen los puntos de vista de cada participante del grupo. Por tanto, uno de los aciertos del trabajo colaborativo que sustentan a la IF, han sido las reuniones directas académico-estudiante y miembros del CAEE-estudiantes, dado que éstas permiten tomar decisiones, asignar tareas, organizar eventos, reportar actividades individuales, comentar asuntos generales, entre otras cuestiones más.

No menos importante es la organización de eventos. Dicha actividad ha requerido del esfuerzo, responsabilidad y disposición de todos, tal como lo señala el entrevistado E1 cuando narra una de las reuniones en las que se discutió sobre la organización de un evento académico:

Sí fue algo bien complicado porque yo me acuerdo que teníamos debates hasta en el nombre de las jornadas o sea, cómo se va a llamar ¿no? Vimos un montón de nombres y todo (...) de mucho diálogo, deliberación y decir bueno... qué nos conviene a todos, qué nos va a resultar, cómo podemos trabajar todos juntos sin tener conflictos y creo que eso fue como lo que nos sirvió y así fue que decidimos el nombre.

Remarcamos aquí la importancia de la comunicación, pero también de la disposición de los integrantes del CAEE para llevar a cabo las tareas que son difíciles y que implican una experiencia, sobre todo para los que nos iniciamos en esas labores.

Podemos en este punto, plantear que entendemos la investigación como un proceso complejo en el que las distintas técnicas de recogida de datos adquieren significado e importancia solamente en relación con los supuestos de las tradiciones intelectuales más amplias en cuyo marco se aplican (Popkewitz, 1988). En cuanto al segundo concepto, formación, partimos de una doble perspectiva. Desde el punto de vista 
del sujeto, Lhotellier (citado por Honoré 1980) la formación "es la capacidad de transformar en experiencias significativas los acontecimientos cotidianos generalmente sufridos, en el horizonte de un proyecto personal y colectivo" (p. 20). Desde el punto de vista de la relación sujeto-objeto según Cooper (citado por Honoré, 1980), formación se entiende como:

La emergencia de un cierto tipo de personas que condensa fragmentos particulares de experiencia, es decir, la superación de contradicción sujeto-objeto, en la medida en que la persona y solamente ella sintetiza estas dos entidades, utiliza activamente los momentos pasivos de su experiencia y observa pasivamente su actividad y la de los demás, hasta el periodo en que la observación llega a ser ella misma acto, y así sucesivamente (p. 20).

En este sentido la formación cobra sentido en la medida en que los alumnos se sienten respaldados por la experiencia de los investigadores y por lo que, tanto a nivel teórico como práctico pueden ofrecer al momento de su participación. Se trata de su actuar en concordancia con sus experiencias y las de los que los rodean, respaldando la formación en la información, siendo estos dos conceptos uno razón del otro. No se puede formar sobre la nada, debe existir un contenido, información sobre la cual podamos construir "algo", es el "pretexto" perfecto para llega a un fin más complejo, la formación.

Por tanto, la investigación formativa se consagra como un espacio de discusión y análisis, es un "proceso abierto" en tanto que posibilita la discusión, el diálogo, el intercambio de posturas y saberes, en consecuencia, rebasa límites y lejos de justificarse en la imposibilidad para actuar, “emprende nuevas búsquedas" (Partido, Figueroa \& Jaramillo, 2009: p. 2).

Se puede decir aquí que obedece al principio "aprender, ayudar, aconsejar, son el orden del cambio, del paso de un estado a otro, bien se trate de un estado físico, afectivo o intelectual" (Honoré, 1980: p. 37), y es que la investigación formativa da paso a nuevas maneras de diferenciar entre lo que educa y lo que forma. En palabras de Honoré (1980):

La educación ha quedado ligada a una concepción jerárquica de la autoridad del educador, de la acción del que conduce a otros mientras éste se deja conducir y por consiguiente a una concepción descendente, desde el que tiene el saber y el poder hasta el que está desprovisto de ellos” (p. 104).

Y la formación es aquella posibilidad para movilizar conocimientos, los cuales "constituyen recursos a menudo determinantes para identificar y resolver problemas, preparar y tomar decisiones" (Perrenaud, 2006, p. 4), tal diferenciación permite subrayar que la investigación formativa, si bien establece jerarquías, no imposibilita a los actores, ni se exenta de reconocer los saberes o habilidades que alguien que no es el coordinador posea.

La investigación formativa, recibe este nombre debido a que es un procedimiento de construcción de conocimientos que sigue los pasos propios del proceso investigativo, con la diferencia de incluir en su práctica actores de diversas disciplinas y grados de formación, lo que enriquece y fortalece al mismo tiempo la construcción de conocimientos.

La estrategia obedece al principio de que "el proceso de apropiación de conocimientos no termina nunca y de que el aprendizaje permanente es condición para la búsqueda de soluciones creativas y para la construcción de nuevas representaciones que hagan posibles nuevas formas de intervención” (Hernández, 2003, p. 187). Además se fundamenta en lo que en palabras De Ibarrola (1989) es que "la mayor y más profunda formación, se adquiere a través del desempeño cotidiano de la profesión y de los límites y posibilidades de desarrollo tanto personal, como del propio campo de investigación, que por lo mismo se logren" (p. 17).

La IF además conlleva una toma de conciencia y fomenta la cultura investigativa, caracterizada ésta última por su creatividad, innovación, 
por el uso de métodos rigurosos, validación y juicio crítico de pares. Constituye la aplicación de hallazgos obtenidos durante el proceso para afinar y mejorar mientras se trabaja, y para servir a los involucrados como medio de reflexión y aprendizaje, incluye un seguimiento de actitudes, creencias, respuestas y asimilación de conocimientos y destrezas al momento de ser ejecutada. La IF deriva en posibilidades de transformación de la práctica en investigación, desarrolla habilidades cognoscitivas: analítica, pensamiento productivo y solución de problemas; familiariza a estudiantes con las etapas de investigación y el planteamiento de problemas; permite un intercambio de saberes no sólo teóricos, sino también prácticos.

Esta estrategia actúa como medio de formación de los actores que la sociedad reclama para trabajar la generación de conocimientos y sus distintas aplicaciones, crea espacios para la práctica, familiariza a los actores con los métodos y técnicas existentes. Al ser considerada como medio, la investigación formativa, implica el aprendizaje de ciertos conocimientos teórico-metodológicos y técnicos del quehacer científico, el fortalecimiento de ciertos valores y principios éticos que se permean en la comunidad científica, así como destrezas, habilidades, actitudes, conducta investigativa, entre otros. Tales elementos constituyen un equilibrio cognoscitivo, epistemológico y metodológico, lo que concluye en una formación para y a través de la investigación.

\section{CONCLUSIÓN}

Haciendo un breve recuento de la experiencia con la implementación de la investigación formativa en la práctica del Cuerpo Académico Estudios en Educación, se puede decir que nace del interés de incluir cada vez un mayor número de estudiantes, quienes al tiempo que apoyan en las actividades de dicho CA, participan en los eventos que organizan (seminarios, diplomados, jornadas, congresos, etc.) se forman y forman a otros, y también son impulsados por los académicos para una participación activa en dichos eventos. La investigación formativa resulta una experiencia colectiva, participan en ella estudiantes y miembros del CAEE que con diversa formación disciplinaria y grado de habilitación académica, participan en un grupo en el que convergen intereses disciplinarios, formativos e incluso personales, que dan vida y nutren el trabajo de un Cuerpo Académico.

Por otro lado, la investigación formativa, al propugnar por la participación de estudiantes; la creación de espacios formativos y de reflexión: actualización y formación constante; integración profesional y personal; y la asignación de tareas, entre otras características más, se consolida como una posibilidad real para cumplir con los requerimientos institucionales sobre el trabajo colectivo, pero también para cubrir aquellas necesidades sociales sobre la producción multi e interdisciplinaria.

Así, la investigación formativa se postula como una posibilidad para atender las exigencias institucionales (trabajo colaborativo), y al mismo tiempo responder a exigencias sociales. Insistimos en las posibilidades que representa desde la experiencia de este Cuerpo Académico para que otros CA y/o grupos de investigación la conozcan, profundicen en ella y la practiquen o desechen a partir de conocer una experiencia, que para este caso ha sido relevante, satisfaciente, de impacto personal y profesional, trascendente y formidable. Misma que ha conllevado el establecimiento de una dinámica de trabajo que se traduce en una dinámica de formación.

\section{REFERENCIAS BIBLIOGRÁFICAS}

De Ibarrola, M. (1989). La formación de investigadores en México. Universidad Futura. 1, (3). México: UAM-Azcapotzalco.

Eguinoa, A. E. (2006). Enseñar a investigar: ¿mito o realidad? Pampedia, (2), 72-78.

Partido, C. M., Figueroa K. L., \& Jaramillo, R., V. (2009). Investigación formativa: una estrategia para la construcción del conocimiento en filosofía, teoría y campo de la educación (FTYCE). Ponencia presentada en el X Congreso Nacional de Investigación Educativa. 
Perrenaud, P. (2006). Construir competencias desde la escuela. Santiago, Chile: Ediciones Noreste.

Hernández, C. A. (2003). Investigación e investigación formativa. Nómadas (18), 183-193.
Honoré, B. (1980). Para una teoría de la formación. Dinámica de la formatividad. Madrid: Narcea.

Popkewitz, T. (1988). Paradigma e ideología en Investigación Educativa. España: Mondadori. 